\title{
MALIGNANT PHYLLODES TUMOR IN A 14 YEAR-OLD PATIENT: A CASE REPORT
}

Leonardo Ribeiro Soares', Joana Ermida Spagnol², Flaviane Marques de Assis², Sebastião Alves Pinto', Alexandre Roriz Blumenschein ${ }^{1}$

${ }^{1}$ Instituto de Mastologia e Oncologia - Goiânia (GO), Brazil.

${ }^{2}$ Faculdade de Medicina, Universidade Federal de Goiás - Goiânia (GO), Brazil.

Introduction: Phyllodes tumor of the breast (PT) is a rare neoplasm, characterized by stromal and epithelial elements, and accounts for approximately $1 \%$ of breast cancer in women. There are three subtypes, according to the World Health Organization, based on histological features: benign, borderline and malignant. Pathogenesis, prognostic and biologic characteristics are unknown, thus the difficulty in assessing the recurrence risk of the tumor. In general, the treatment approach is breast conserving surgery, with a margin $>1 \mathrm{~mm}$, in order to prevent recurrence. There is currently a great concern with the increase of breast cancer cases in young women, which represents a significant burden in developing countries such as Brazil. The mean age of presentation of malignant PT is 40 years old, and there are rare cases reported in very young adults, 18 and 23 years old. There is one case series in the literature of benign PT in 14 to 16 years old adolescents. However, we did not find any report of malignant PT in this age group. Given the rarity of the case and the current importance of breast cancer in young women, we report a rare case of malignant PT in a patient with only 14 years of age. Case report: A 14-year-old female came to a visit due to an abnormal growth of the breast over the last nine months. She had no other complaints or comorbidities. In her family history, she has one aunt that had breast cancer. On physical examination, the patient presented gigantomastia and stretch marks stretch with a reddish appearance. It was identified a nodule of $5.0 \mathrm{~cm}$ located in the upper inner quadrant of the right breast, and the axillary region showed no abnormalities. On the ultrasound, the nodule measured $5.7 \mathrm{~cm}$. The patient underwent a segmentary resection of the lesion. The anatomopathological study revealed a malignant PT with a low differentiation grade, measuring $7.0 \mathrm{~cm}$ in its largest dimension and free margins, however exiguous. Immunohistochemistry of the lesion revealed the expression of Ki-67, p-63 protein, cytokeratin-14, Bcl-2 and vimentin. On the magnetic resonance imaging, only surgical alterations were observed with no macroscopic residual tumor and the armpit was negative. In order to extend the surgical margins, a second approach was performed, associated with a reduction mammoplasty. The anatomopathological study then showed no residual neoplasm and free margins. The patient had good postoperative recovery and was satisfied with the esthetic results. After consultation with the oncologist, no adjuvant treatment was indicated. 